\title{
La grande transformation des PME japonaises
}

\section{Le rôle central des compétences}

Au lendemain de la Deuxième Guerre mondiale, l'économie japonaise est caractérisée par une structure duale, avec de fortes disparités entre les grandes et les petites entreprises. Les PME sont alors considérées comme une menace pour I'industrialisation du Japon. Aujourd'hui elles en sont une des forces principales et sont perçues comme un moteur de croissance pour les années à venir. Cet article étudie la transformation opérée par les PME japonaises au cours des cinquante dernières années et met en évidence les facteurs qui l'ont facilitée: la sous-traitance, les caractéristiques inhérentes aux PME, les réseaux et les districts industriels. La politique industrielle n'a finalement eu qu'un impact très limité et ne constitue qu'une explication partielle de la montée en puissance des PME. Enfin, l'article évoque la délocalisation industrielle et montre comment elle modifie les sources d'acquisition des compétences des petites entreprises. 
u lendemain de la Deuxième
Guerre mondiale les grandes entre-
prises japonaises ont connu une croissance très forte et se sont imposées comme les leaders de la reconstruction économique et industrielle, laissant les PME loin derrière. Dans les années 1950-1960, la différence entre les grandes et les petites entreprises est telle que l'économie est qualifiée de « duale » par l'administration nationale (Ministry of International Trade and Industry, 1957). Le retard des PME est alors ressenti comme une menace pour la modernisation du Japon et contribue à façonner l'opinion selon laquelle la structure duale est un problème économique important. Ainsi, le Economic White Paper de 1957 (MITI,
1957) indique que « Le plein emploi, qui est l'objectif final de l'économie japonaise, va non seulement permettre de diminuer le nombre de chômeurs mais il va aussi dissoudre la structure duale à travers la modernisation et la croissance de l'économie. » (p. 36-37). Les écarts entre les deux éléments constitutifs de l'outil productif japonais sont effectivement très importants. Les caractéristiques les plus visibles de ce problème de structure duale sont les différences de productivité et de niveaux de salaires entre les grandes et les petites entreprises. De plus, les petites entreprises industrielles ressemblent plus à des magasins artisanaux qu'à de véritables usines et elles ont un équipement basique et obsolète (Itami, 1998).

Figure 1 - L'image des PME japonaises

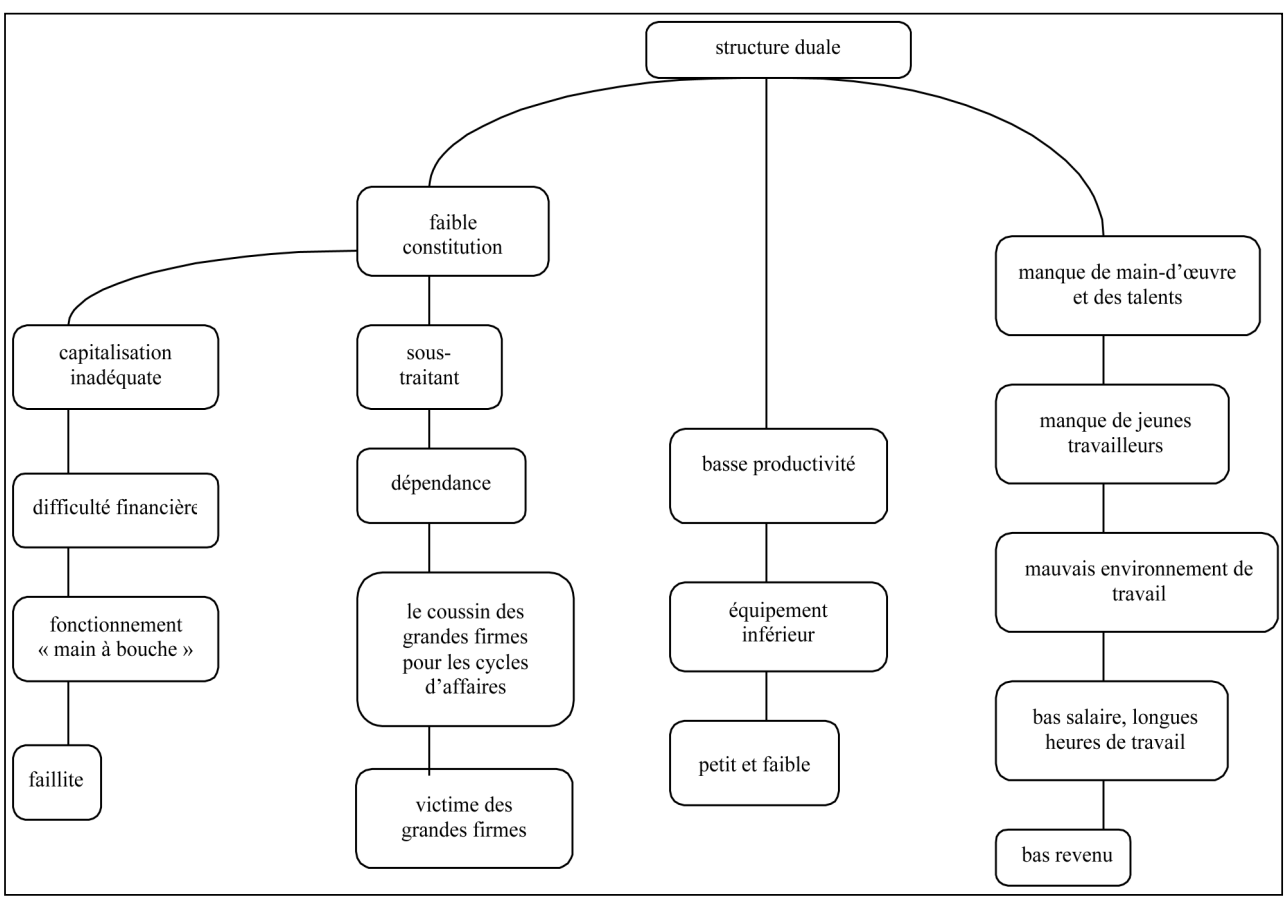

Source: adapté de Small Business White Paper, MITI (1972, p. 259).

Note: Fonctionnement « main à bouche » signifie que ce qui est produit par l'entreprise sert essentiellement à « nourrir » l'entrepreneur. 
L'image des PME dans ce contexte a été présentée dans une enquête réalisée par le MITI (Small Business White Paper, 1972) (figure 1). Elle met en évidence les problèmes au niveau de la situation financière, de la dépendance, de la productivité et de la main-d'œuvre.

La comparaison de cette image avec celle d'aujourd'hui est surprenante. Aujourd'hui, les PME manufacturières possèdent un équipement moderne et des compétences importantes (SME Agency, 2002). Elles produisent des biens de qualité, investissent dans la recherche et le développement, développent leurs propres produits. Certaines sont devenues des leaders mondiaux dans leur domaine. C'est par exemple le cas de Hyoda ou de Japan Remote Control Company, entreprises installées à Higashi Osaka, dans les domaines des thermomètres industriels et des instruments de mesure pour les hélicoptères. Le gouvernement japonais considère aujourd'hui que les PME sont l'un des moteurs de la croissance économique (MITI, 2000 ; SME Agency, 2002; METI, 2002). De plus, les politiques économiques et industrielles récentes qui visent la mise en place des clusters $^{1}$ industriels et des clusters de connaissances $^{2}$ reposent sur les forces et les compétences des PME, qui devraient être renforcées par des partenariats avec des universités et des centres de recherche. $\mathrm{La}$ situation a donc drastiquement changé et le Japon, comme d'autres pays développés, reconnaît pleinement l'importance des
PME (OCDE, 1996; OECD, 1999, 2001). Il semble que « le problème des PME » se soit transformé en une force et que la question de l'économie duale ne soit plus d'actualité. Comment peut-on expliquer ce changement? Comment les PME ont-elles réussi à surmonter leurs insuffisances pour devenir des acteurs reconnus de l'industrie et de l'économie? Cet article étudie la transformation des PME japonaises et discerne les facteurs qui ont été à l'origine de celle-ci. Différents courants de la littérature font émerger les facteurs expliquant la montée en puissance des PME japonaises. Ces facteurs sont:

1) la sous-traitance pour les grandes entreprises (Asanuma, 1989) ;

2) les caractéristiques et la nature des PME (Kiyonari, 1972; 1990) ;

3) le networking, les réseaux et la concentration dans des districts industriels (Itoh et Urata, 1994; Seki, 1994).

Le premier et le troisième facteur sont liés à des éléments à la fois internes et externes aux entreprises, alors que le deuxième est d'ordre interne. Ces trois groupes de facteurs ont, chacun, permis aux PME de générer, d'accumuler et d'améliorer leurs compétences. De fait, nous montrons dans cet article que l'acquisition de nouvelles compétences a joué un rôle majeur dans la transformation des PME japonaises, les mesures politiques ayant plutôt suivi que guidé la transformation des petites entreprises.

Nous examinons d'abord les trois facteurs de progrès des PME. Dans un deuxième

1. Nous utilisons le mot anglais cluster pour désigner les concentrations industrielles associées à des nouvelles technologies et au sein desquelles les universités ou les centres de recherche jouent un rôle important. C'est en cela qu'elles se différencient des districts industriels.

2. Industrial Cluster Plan du METI (le MITI est devenu le METI - Ministry of Economy, Trade and Industry en 2001) et Knowledge Cluster Plan du ministre de l'Éducation, des Sports, de la Culture et des Sciences et Technologies - le MEXT. 
temps, nous nous intéressons à la politique industrielle et montrons qu'elle n'a eu qu'une portée limitée pour les PME. Enfin, nous discutons des évolutions récentes dans l'industrie japonaise et examinons la viabilité des trois facteurs de progrès dans les années à venir.

\section{LES FACTEURS DE PROGRÈS}

Comme nous l'avons évoqué précédemment, l'acquisition de compétences a été à l'origine du progrès des PME au cours des cinquante dernières années. Analysons maintenant les facteurs qui l'ont facilité.

\section{La sous-traitance: dépendance et apprentissage}

Le premier facteur qui a contribué au progrès des PME est la sous-traitance pour les grandes entreprises. Ce n'est pas uniquement la sous-traitance en tant que telle qui a engendré ce progrès mais plutôt un ensemble d'éléments qui y sont associés.

La sous-traitance au Japon, de la même manière que des phénomènes liés tels que les liens particuliers entre les entreprises connus sous le nom de keiretsu ou la mise en place de nouvelles techniques de gestion de la production comme le juste-à-temps, ont fait l'objet de nombreuses études dans les pays occidentaux (Schonberger et Gilbert, 1983; Carillon, 1986; Hay, 1988). Le Japon est en effet devenu dans les années 1980 un modèle d'efficacité en termes d'organisation de la production. $\mathrm{La}$ sous-traitance est au cœur de ce système et elle est considérée comme la clé de la réussite de l'économie japonaise après la Deuxième Guerre mondiale (Dore, 1986, 1987; Asanuma, 1989; Fruin, 1992; Gerlach, 1992; Morris et Imrie, 1992; Sako, 1992; Ito et Rose, 1994; Lung, 1999). Elle a été décrite comme le type idéal d'organisation de la production ${ }^{3}$. Ainsi, dans les années 1980, il est devenu communément admis que les Japonais avaient atteint des niveaux de performance extraordinaires grâce aux liens de sous-traitance entre les entreprises et grâce à la mise en place de techniques de gestion efficaces. De nombreuses études ont porté sur ces méthodes d'organisation de la production, mais peu d'entre elles ont mis en avant le rôle et le mérite des PME sous-traitantes qui y participent. Or, ces systèmes n'auraient pu se mettre en place si les grandes entreprises n'avaient pu faire parfaitement confiance aux PME sous-traitantes, éléments intégrants de tels systèmes.

Dans les années 1950 et suite à la dissolution des grands groupes d'avant-guerre, appelés zaibatsu, les grandes entreprises s'organisent en keiretsu - des groupements d'entreprises entretenant des liens très étroits. Les keiretsu ont repris le rôle des zaibatsu d'avant-guerre en tant que pôles de développement économique. La forte concurrence entre les PME les a obligées à entrer dans des relations de sous-traitance et ainsi à faire partie des keirets $u^{4}$ même si les paiements, les délais de livraison et les prix

3. Comme le souligne Lung (1999, p. 229): "All of the interpretative grids being used (Toyota Production System, Ohnoism, Toyotism, Lean Production, etc.) emphasize the organization of sub-contracting, as an 'ideal-type' and a benchmark."

4. Les PME faisaient partie des keiretsu dans la mesure où elles sous-traitaient uniquement pour une entreprise. Dans certains cas, les grandes entreprises possédaient des parts des PME, mais ceci n'était pas un phénomène généralisé. 
imposés par les grandes entreprises étaient très contraignants. Comme le souligne Matsushima (2001, p. 12), « la relation de sous-traitance a été principalement celle des keiretsu où la PME dépend absolument de son client pour son existence. »

Un des éléments centraux de la relation de sous-traitance est l'engagement contractuel basé sur le sentiment d'obligation (appelé obligational contract). C'est le contrat de sous-traitance le plus fréquemment rencontré au Japon. Le système d'engagements contractuels a commencé à se développer pendant la période de forte croissance des années 1960, à l'époque où de nombreuses grandes entreprises augmentaient leurs volumes de production et avaient besoin de l'aide des entreprises sous-traitantes. En conséquence, les grandes entreprises clientes ont été incitées à mettre en place des contrats exclusifs d'approvisionnement pour empêcher que les compétences développées chez leurs fournisseurs ne soient diffusées aux concurrents. Cela a mené graduellement à la création de structures pyramidales. Parallèlement, les fournisseurs étaient sous pression pour améliorer leur technologie et pour développer des processus de production sophistiqués à haute valeur ajoutée. Une conséquence a été la dépendance croissante des grands producteurs vis-à-vis de leurs fournisseurs pour le travail d'assemblage, d'approvisionnement des pièces mais aussi pour le design. En même temps, de nombreux fournisseurs ont développé leurs propres produits et sont ainsi devenus plus indépendants et plus concurrents (Asanuma, 1989; Gerlach, 1992 ; Whittaker, 1997).

Dans ce cadre, les liens entre les donneurs d'ordre et les sous-traitants sont de longue durée, basés sur la confiance et l'obligation mutuelle. La métaphore entreprise-parent entreprise-enfant ou simplement parentenfant est souvent utilisée pour désigner des liens étroits entre les constructeurs et leurs sous-traitants. La sous-traitance japonaise est caractérisée par des liens hiérarchiques, l'existence de niveaux ou de couches de sous-traitants qui forment une « pyramide » au sommet de laquelle se trouve le donneur d'ordre. La base de la pyramide est formée par de très nombreuses PME, dont beaucoup sont des micro-entreprises (Sako, 1992). La grande entreprise, l'entreprise principale, communique avec les fournisseurs du premier niveau et permet à ceux-ci de communiquer avec les fournisseurs du deuxième niveau et ainsi de suite. En général, l'entreprise principale ne connaît pas les petits sous-traitants qui se trouvent en bas de la pyramide. Pour que l'intégration effective de ce type d'organisation de la production soit maintenue, la coordination dans les activités de design, de conception des produits et de R\&D est encouragée, favorisant l'association à long terme. Les liens contractuels entre les entreprises sont donc majoritairement de longue durée (Asanuma, 1989; Gerlach, 1992; Morris et Imrie, 1992; Sako, 1992; Nishiguchi, 1994).

Les relations de sous-traitance sous-entendent la signature d'une série de contrats. $\mathrm{Au}$ début de la relation, le sous-traitant signe un contrat commercial de base qui met l'accent sur la sincérité et la bonne foi. Des clauses de confidentialité et de secret peuvent aussi être exigées. Le contrat final concerne les spécifications du travail, les délais et les prix, qui sont déterminés après l'estimation des coûts. Une fois le contrat signé, le sous-traitant ne peut plus changer les conditions en sa faveur, et surtout les 
prix, même si les spécifications des produits ou du travail à effectuer changent. C'est sa seule responsabilité d'organiser le travail, il est libre d'utiliser des sous-traitants ou d'effectuer seul tout le travail. Les objectifs du sous-traitant sont de diminuer les coûts et les prix et de répondre aux exigences du donneur d'ordre, préservant ainsi la relation à long terme (Sako, 1992). L'accent sur la loyauté du sous-traitant permet d'éviter les conflits devant être réglés en justice et de lutter contre les comportements opportunistes. Les donneurs d'ordre attendent de leurs sous-traitants de réduire progressivement les coûts à chaque fois que les prix unitaires sont révisés, ce qui arrive habituellement deux fois par an. En même temps qu'ils sont intéressés par les prix, les clients sont aussi intéressés par les capacités technologiques des fournisseurs et sont prêts à soutenir leur activité. Dans certains cas, les donneurs d'ordre aident le soustraitant à surmonter une crise financière. La décision de donner ou non une aide financière au sous-traitant dépend du niveau de profit qui peut être retiré et de l'historique de la relation (Whittaker, 1997).

Pour les grandes entreprises, la sous-traitance permet de produire des volumes plus importants et de transférer le risque des affaires vers la périphérie, les PME. De plus, les grandes entreprises sont capables de produire plus de modèles, de raccourcir le temps de design et de développement des nouveaux produits, dans la mesure où elles peuvent se concentrer sur ces activités plutôt que sur la production elle-même. Les grandes entreprises travaillent sur le développement des nouveaux équipements, plus modernes, elles acquièrent de nouvelles méthodes, en inventent d'autres et appliquent des méthodes de gestion de plus en plus efficaces. Un exemple typique est le juste-à-temps développé par Toyota. Presque tous les grands constructeurs japonais ont adopté soit le juste-à-temps, soit des méthodes qui reposent sur les mêmes principes, comme par exemple le « système synchro » de Nissan (entretien à Tachi-S, le premier sous-traitant de Nissan, 2003). Dans le système juste-à-temps, les assembleurs finaux sont soutenus par des sous-traitants qui livrent les pièces selon des délais très précis et suivant les spécifications comprenant les objectifs, les prix et les termes de livraison (Nishiguchi, 1994). Cette méthode impose une réactivité importante de la part des fournisseurs car les pièces nécessaires doivent être livrées dans des délais très courts. Toutefois, il est important de noter que c'est uniquement parce que les PME sous-traitantes ont pu répondre à ces exigences que de tels systèmes ont pu être mis en place. Sans leur coopération et leur aptitude à adopter ce nouveau style de gestion de production, les constructeurs n'auraient pas pu les envisager.

La sous-traitance et l'appartenance aux keiretsu imposent des conditions difficiles aux PME. En effet, elles doivent constamment diminuer leurs coûts, améliorer la qualité des pièces, produire dans des délais très courts, accepter des délais de paiement relativement longs. De plus, bien que les contrats entre les entreprises soient de long terme et reposent sur des engagements mutuels, la concurrence entre les sous-traitants est entretenue par les donneurs d'ordre, car un sous-traitant peut être remplacé en cas de baisse de compétitivité. Ces conditions difficiles obligent les PME à se remettre en cause constamment, à améliorer leur qualité, à devenir plus efficaces, plus 
flexibles et à s'adapter plus facilement à des exigences de plus en plus importantes (Sako, 1992). Le sous-traitant cherche à atteindre des standards élevés de production car cela lui permet de gagner une bonne réputation aux yeux du client.

Les PME sont donc soumises à des contraintes fortes de la part des grandes entreprises, mais bénéficient aussi de relations à long terme, qui réduisent les risques et permettent un planning plus sûr. Les sous-traitants apprennent beaucoup et d'autant plus qu'ils bénéficient d'un transfert de technologie du « haut vers le bas »- les donneurs d'ordre leur transmettent des nouvelles techniques, leur montrent quel équipement acquérir et comment améliorer leur technicité et leur productivité. Autrement dit, les PME sous-traitantes bénéficient d'un véritable apprentissage et se rapprochent de plus en plus de leurs donneurs d'ordre en matière de production, de technologie, d'efficacité et de performance. Selon Aoki (1988), les avantages mutuels des relations à long terme entre les donneurs d'ordre et les sous-traitants ont pour résultat une quasi-rente relationnelle.

Ainsi, grâce à cette position de sous-traitants, les PME deviennent de plus en plus performantes, leur production est de plus en plus moderne et efficace, la qualité de leur travail s'améliore. Les PME sont capables de faire face à des exigences constantes de leurs clients. De plus, elles acquièrent, accumulent et améliorent leurs compétences.

\section{Les caractéristiques inhérentes aux entrepreneurs}

Le deuxième facteur à l'origine des progrès importants des PME repose sur leurs caractéristiques propres. En effet, les traits majeurs des PME leur ont permis d'évoluer, alors qu'au départ elles n'étaient pas dotées de ressources et de compétences particulières. Ces caractéristiques existaient par le passé mais c'est après la Deuxième Guerre mondiale et la prolifération des petits établissements manufacturiers qu'elles se sont affirmées.

La première de ces caractéristiques est un esprit d'entreprenariat très fort (Kiyonari, 1990), et la fierté de mener sa propre affaire. Ceci est d'autant plus significatif que les conditions de vie et de travail dans les grandes entreprises au Japon sont bien meilleures que celles au sein des PME. Par conséquent, pour qu'une personne choisisse ce mode de vie difficile, avec moins de sécurité et un temps de travail très long, il faut qu'elle ait un esprit entrepreneurial fort. En effet, les entrepreneurs japonais ont souvent des personnalités très fortes, ils n'acceptent pas de rester des salary $m e n^{5}$ ordinaires (Seki, 1994; Storz, 1999). La motivation qui incite les entrepreneurs à se lancer dans la création de leurs propres entreprises est leur volonté de devenir « maître de leur propre château » (ikkoku ichijo no aruji) ou de "lever leur propre drapeau » (hitohata o ageru). On retrouve ici l'idée féodale d'attachement aux petites affaires avec l'intention de les transférer à

5. Les « cols blancs » japonais sont ainsi dénommés. En japonais, cette expression est prononcée sarari man. 
la famille, une forme d'héritage. Ce point est généralement négligé dans les analyses de la culture industrielle japonaise, mais il est fondamental pour comprendre l'entreprenariat (Kiyonari, 1990; Whittaker, 1997). En effet, la philosophie de « maître de son château » est un type d'individualisme qui est contradictoire avec l'image groupiste, typique de la société japonaise (Hashimoto, 1989; Kiyonari, 1990). Toutefois, il ne s'agit pas d'un individualisme atomistique, au contraire - il s'est développé le plus dans le cadre de la coopération dans des grands districts industriels.

Dore (1986) avance qu'il existe un lien entre, d'une part, les PME dans le secteur manufacturier et, d'autre part, l'agriculture. Avant la Deuxième Guerre mondiale, l'agriculture japonaise était une activité familiale à petite échelle et les agriculteurs devaient posséder des compétences managériales, qui impliquaient les achats des produits nécessaires à la production, la gestion des ventes et des revenus. Pendant l'industrialisation, ces compétences ont été véhiculées dans les zones industrielles, soit par des paysans qui ont gardé des liens avec l'agriculture et développé parallèlement une affaire industrielle, soit par leurs enfants qui ont apporté des attitudes rurales dans les zones industrielles. L'image commune de l'origine des fondateurs de petites entreprises est qu'ils sont issus de familles agricoles.

Une autre force des PME, liée à l'esprit entrepreneurial, est l'effort constant pour améliorer les compétences, pour toujours se dépasser. La production est une fierté pour les entrepreneurs. Ils ont en effet une véritable « culture de la production " (Seki, 1994). Avoir des compétences éle- vées dans la production est considéré comme l'un des principaux atouts de l'entreprise. Ainsi, il y a par exemple des petites usines qui produisent des moules (à partir desquels des milliers de produits sont fabriqués) avec une précision qu'aucune grande entreprise ne serait capable de reproduire. Cette quête du dépassement des compétences est liée à un esprit d'artisanat qui a survécu malgré l'adoption de nouvelles techniques de production et l'utilisation des machines à commande numérique.

Certains entrepreneurs créent leurs entreprises pour réaliser un nouveau produit et développer un équipement original. L'innovation peut être la cause du départ des salariés des grandes entreprises pour établir leur propre affaire (Kiyonari, 1990). Les entrepreneurs ont souvent travaillé dans deux ou trois entreprises avant de lancer leur propre affaire.

Une autre caractéristique importante des PME japonaises est la façon dont les entrepreneurs mélangent la vie privée et la vie professionnelle. Une fois l'entreprise établie il faut la maintenir et la développer. Pour cela les entrepreneurs travaillent dur et ont des volumes horaires de travail importants. Pour réduire leur temps de déplacement, ils habitent plutôt dans la proximité, souvent à côté ou au-dessus des locaux de production (Kiyonari, 1990 ; Seki, 1994). Le travail et la vie privée ne sont pas clairement séparés, ni physiquement, ni psychologiquement. Le travail est pour beaucoup d'entrepreneurs la sphère de vie (seikatsu no ba) (Kiyonari, 1990). C'est un élément majeur de leur identité et de leur estime personnelle. Le travail ne peut se réduire simplement à un calcul économique. Preuve en est la volonté des entrepreneurs de garder 
l'entreprise même lorsqu'elle n'est pas économiquement viable.

$\mathrm{Au}$ niveau relationnel, un trait majeur des entrepreneurs est le sentiment paternaliste vis-à-vis de leurs employés. Contrairement aux grandes entreprises, qui sont en mesure d'offrir aux employés la sécurité de l'emploi, des conditions et des salaires supérieurs, les PME sont associées à l'exploitation, à l'insécurité et à la difficulté du travail. La mauvaise image du travail dans les PME est résumée au Japon par les « 3K »-kitanai, kiken, kitsui - qui signifient sale, dangereux et difficile. Il ne faut toutefois pas sous-estimer les avantages qui peuvent compenser en partie ces mauvaises conditions: l'apprentissage, l'atmosphère familiale et la possibilité, pour les salariés, de devenir indépendants (Whittaker, 1997; Storz, 1999). L'acquisition de nouvelles compétences et l'apprentissage sont donc des motifs pour lesquels les employés choisissent les PME. La question des compétences, l'attraction et la motivation des jeunes ouvriers constituent un défi critique pour les petites firmes manufacturières. Pour répondre à ce défi, les entrepreneurs doivent innover, non seulement en termes de machinerie mécanique mais aussi en termes de conditions et d'environnement de travail.

Les entrepreneurs font tout leur possible pour rester en contact avec leurs employés même lorsque ces derniers décident de partir et de créer leurs propres entreprises. Une pratique courante est la mise en place d'une relation donneur d'ordre-sous-traitant avec les anciens employés. Souvent, les patrons aident leurs employés à lancer leur propre affaire ou deviennent leurs donneurs d'ordre (Seki, 1994; Storz, 1999).
Les liens personnels sont traditionnellement importants pour rassurer les ouvriers. Les liens de parenté et les liens de voisinage assurent que la relation d'emploi est plus qu'un échange travail contre argent. Beaucoup de propriétaires ont un fort sentiment de responsabilité vis-à-vis de leurs employés et de leurs familles. Par le passé, les employés attendaient du propriétaire de leur trouver une femme et de financer l'équipement scolaire de leurs enfants. Des relations étroites de ce genre ont constitué des sortes de substituts aux salaires plus élevés et aux meilleures conditions qu'offrent les grandes entreprises. La gestion du personnel ne peut aucunement être qualifiée de gestion systématique. C'est plutôt un mélange de sentiments paternalistes, d'autorité singulière et de recherche constante de compétences et d'accomplissement des tâches et des ordres (Whittaker, 1997). Dans les années 1960, avec l'apparition du manque de main-d'œuvre au Japon, le paternalisme a partiellement perdu sa force d'attractivité des salariés dans les PME. Il continue toutefois à jouer un rôle.

Ces caractéristiques inhérentes aux PME japonaises et surtout à leurs entrepreneurs, à savoir l'esprit entrepreneurial, la volonté de se dépasser, l'implication totale dans l'entreprise et les liens paternalistes entre employeurs et employés, ont constitué un des facteurs de progrès des PME japonaises au cours des quarante dernières années.

\section{La concentration dans les districts industriels, le networking et les réseaux}

Le troisième facteur qui a joué un rôle dans le progrès des PME depuis les années 1960 est la concentration géographique des PME manufacturières et leur propension à créer des liens entre elles. 
Les PME manufacturières japonaises ont tendance à s'agglomérer dans des zones géographiques restreintes, formant ainsi des concentrations de plusieurs centaines, voire plusieurs milliers d'entreprises. Ces concentrations, appelées districts industriels, sont un phénomène typique de l'industrie japonaise (Itoh et Urata, 1994). Il y a au Japon 550 districts industriels (MITI, 2000) dans des industries comme le textile, la machinerie, le métal, la céramique et autres. Le rôle le plus connu des agglomérations industrielles japonaises est la formation de réseaux hiérarchiques de soustraitance, comme une partie du système de keiretsu. Dans les districts industriels de type « ville-entreprise » ou « villechâteau » comme Hitachi-city ou Toyotacity, les réseaux géographiques forment des structures hiérarchiques. Toutefois, d'autres districts se caractérisent par des réseaux de type many-to-many, autrement dit des réseaux prenant une forme plus horizontale au sein desquels les entreprises ne dépendent pas d'un seul client pour leurs ordres. Dans des agglomérations où l'on trouve un mélange d'industries variées, les réseaux horizontaux ont tendance à être encastrés historiquement dans la région (Colovic et Tayanagi, 2004).

Malgré l'intérêt considérable des chercheurs occidentaux pour les districts industriels (Becattini, 1987; Piore et Sabel, 1984; Benko et Lipietz, 1996, 2000), la connaissance de ces districts au Japon est très limitée (Whittaker, 1997). Toutefois, la vitalité des concentrations de petites entreprises et en particulier de celles de Tokyo ${ }^{6}$, a attiré un intérêt important des chercheurs japonais (Sato, 1981; Seki, 1994 ). Ils ont étudié la diversité de la base industrielle et la façon dont ces «zones complexes » ont évolué à travers le perfectionnement des capacités techniques des petites entreprises, liées par des liens horizontaux. Seki (1994) par exemple, évoque des entreprises qui se mêlent les unes aux autres dans la même région, formant ainsi un regroupement important de technologies. Entre les usines il y a une sorte de " commerce confrère ». Les petites entreprises comptent sur les grandes pour avoir des commandes régulières mais passent aussi beaucoup de commandes les unes aux autres (Whittaker, 1997). Le commerce confrère est accompagné d'une rivalité « confrère ", d'une concurrence soutenue par un encouragement mutuel et d'une assistance mutuelle très développée. Les entreprises travaillent ensemble à chaque stade de la production, permettant la production de très petits lots et l'implantation de tous les procédés de fabrication. Elles ont souvent des ressources et des compétences interconnectées. Bien que chaque entreprise soit spécialisée dans un domaine d'expertise très étroit, elles possèdent collectivement toutes les compétences dans un domaine donné. En fonction de leur localisation et des caractéristiques productives, quatre types majeurs de districts industriels peuvent être distingués : les villes-entreprises, les zones industrielles urbaines, les districts régionaux et les districts géographiquement basés ou traditionnels (Japanese Small Business Research Institute - JSBRI,

6. En raison des compétences et de la spécialisation des PME, de la position centrale de Tokyo et de la proximité des concentrations industrielles avec les centres de recherche, les concentrations industrielles situées dans la capitale japonaise ont fait l'objet de plusieurs études. 
1998). Au sein des villes-entreprises comme Toyota ou Hitachi, une grande entreprise place les ordres auprès des PME locales. Les zones industrielles urbaines sont typiquement caractérisées par l'absence d'unique donneur d'ordre et par leur localisation au sein des grandes villes, comme Tokyo ou Osaka. Les districts régionaux sont des centres industriels où différentes industries peuvent être présentes. Enfin, les districts géographiquement basés ou traditionnels se consacrent à des industries de produits de consommation, comme le textile et la céramique.

Les concentrations industrielles sont nombreuses et variées et elles contiennent de multiples petites entreprises manufacturières. Au sein des districts industriels, les PME sont en mesure d'avoir des échanges intenses les unes avec les autres car le networking est facilité par la proximité géographique.

Le networking est une activité très répandue au Japon, pour les grandes et les petites entreprises, de même que pour la société dans son ensemble. Les personnes qui connaissent beaucoup de monde sont particulièrement appréciées. Les japonais utilisent la métaphore " avoir un visage large » (kao ga hiroi) pour désigner les personnes qui ont beaucoup de connaissances. Quand il s'agit des PME, le networking est d'autant plus développé que ce sont à la fois les entrepreneurs qui ont des relations avec d'autres entrepreneurs, mais aussi les ouvriers qui connaissent d'autres ouvriers dans les entreprises voisines (Sato, 1981; Seki, 1994; Whittaker, 1997). Les liens sont donc créés tant au niveau des propriétaires et dirigeants qu'à celui des employés. Les entrepreneurs japonais s'engagent souvent dans des relations de réseau et leurs entreprises entrent dans des activités de coopération les unes avec les autres. Cette coopération peut prendre différentes formes : la production jointe d'une pièce ou d'un produit, la R\&D, le partage d'ordres, le partage d'équipements ou d'informations. Les réseaux peuvent être de type vertical ou horizontal, formel ou informel. Ils permettent aux entreprises d'acquérir de nouvelles connaissances qui, elles-mêmes sont en mesure d'engendrer de nouvelles compétences ou d'améliorer les compétences existantes. C'est en effet au sein des relations avec les autres entreprises que les PME bénéficient d'un véritable apprentissage.

Ainsi les entreprises manufacturières s'organisent avec les entreprises environnantes et forment des relations multiples. Elles sont encastrées dans leur environnement. Ces toiles riches de relations sont le plus facilement développées au sein de nombreux districts industriels, en raison de la haute concentration d'industries et de la proximité physique d'unités de production. Les interactions et la coopération avec les entreprises voisines permettent aux PME d'acquérir des nouvelles connaissances et d'améliorer les compétences existantes.

La sous-traitance, les caractéristiques endogènes des PME, les réseaux et les districts ont ainsi, tour à tour, contribué à la constitution d'un portefeuille de compétences et de connaissances. Ces connaissances ont permis aux petites entreprises japonaises de surmonter leurs points faibles et de se transformer en un acteur majeur de l'économie japonaise. On peut alors s'interroger sur le rôle qu'a occupé la politique industrielle dans la transformation des PME japonaises. Pourrait-elle représenter une explication alternative à celle proposée? Dans la sec- 
tion suivante nous montrons qu'elle n'a eu qu'une influence limitée sur la transformation des PME japonaises.

\section{QUEL A ÉTÉ LE RÔLE DE LA POLITIQUE INDUSTRIELLE?}

Il a souvent été souligné qu'au Japon l'État ait un rôle de guide très important pour les entreprises. Le rôle du MITI a été particulièrement mis en avant (Dore, 1986; Ito, 1992 ). Se pose alors la question de savoir si la politique industrielle orientée vers les PME est une explication alternative de leur succès. Plusieurs éléments semblent indiquer que cette piste est à nuancer.

Tout d'abord, certaines recherches ont suggéré que le gouvernement japonais n'a jamais pris de décisions de politique industrielle très fortes. Ainsi, la capacité sans précédent du MITI à soutenir la forte croissance est définie comme un " consensus » (rolling consensus) (US Department of Commerce, 1972), « minimaliste plutôt que dirigiste » (minimalist rather than dirigist), " en coordination parfaite " (in perfect coordination) (Okimoto, 1989) entre le Gouvernement (MITI) et l'industrie. Autrement dit, selon ces recherches, les actions gouvernementales accompagnaient plutôt qu'elles ne guidaient l'industrie.

D'autres chercheurs ont souligné le fait que parmi les domaines non visés par le MITI, comme les appareils photographiques, les machines à coudre, les bicyclettes, les motocycles, les radios, les téléviseurs, les calculatrices, les machine-outils, beaucoup ont connu un développement important (Ito, 1992). L'exemple de Honda (moto- cycles) et de son entrée aux États-Unis (Pascale, 1984) contre le gré du MITI montre que cette institution japonaise avait une portée limitée. Un deuxième exemple significatif est celui de l'industrie automobile. Dans les années 1950-1960, le MITI a estimé que le Japon, étant un « petit pays », n'avait besoin que d'un ou deux producteurs d'automobiles. Cependant, les constructeurs d'automobiles ont lutté contre les pressions de MITI et beaucoup d'entre eux sont aujourd'hui parmi les plus grands constructeurs mondiaux.

La politique des PME industrielles a également été jugée comme ayant eu une portée limitée. Comme le met en avant Miwa (1996) en citant Hajime Takaki, l'ancien président de la Banque centrale coopérative de commerce et d'industrie du Japon:

« Il y a tellement de mesures politiques différentes pour les petites entreprises que la plupart d'entre nous serions stupéfaits de découvrir la petite échelle de chacune d'entre elles. Les efforts politiques ont été importants mais très superficiels. C'est comme si le gouvernement arrosait des grands pots de fleurs avec de tous petits arrosoirs. (...) Chacune des politiques est bien conçue et pertinente, mais l'échelle des politiques gouvernementales est trop petite pour avoir quelque effet que ce soit. ».

De plus, si l'on compare la politique industrielle qui visait les PME et les conditions réelles des PME à différentes périodes on s'aperçoit que cette politique ne faisait que suivre la situation sur le terrain (Colovic et Tayanagi, 2004) ${ }^{7}$. Ceci était particulièrement vrai jusqu'au début des années 1990.

7. Pour en savoir plus sur la politique visant les PME au Japon, voir Miwa (1996), Matsushima (2001) ou Colovic et Tayanagi (2004). 
Les mesures gouvernementales étaient plus orientées vers la protection des PME de la concurrence extérieure (notamment dans les années 1980) que vers l'incitation à l'acquisition des nouvelles compétences. Ainsi, il existait un écart entre la politique gouvernementale et les motivations des PME. Le progrès des PME japonaises ne peut s'expliquer que très partiellement par l'efficacité de la politique industrielle.

En conclusion, les PME japonaises ont réussi à surmonter leur retard par rapport aux grandes entreprises grâce à trois facteurs: la sous-traitance pour les grandes entreprises, les caractéristiques inhérentes aux PME et le fonctionnement en réseaux. Le retard a pu être rattrapé grâce à la création, l'amélioration et l'accumulation des compétences. Ce sont donc ces trois facteurs qui ont constitué la source de la création des compétences pour les PME.

Ces éléments pourront-ils, et dans quelle mesure, continuer à générer la construction du « capital compétences » des PME japonaises? La section suivante étudie la situation actuelle des PME au Japon et examine la viabilité de ces facteurs dans les années à venir.

\section{L'IMPACT DES DÉLOCALISATIONS}

Les PME japonaises possèdent à l'heure actuelle un véritable capital de compétences dans tous les secteurs industriels. Toutefois, pour continuer à occuper une place-clé dans le système économique, elles doivent constamment travailler sur le renforcement de ce capital. Autrement dit, elles ne peuvent pas se limiter à leurs compétences présentes pour répondre aux besoins futurs. Leurs compétences doivent suivre les évo- lutions technologiques et leur permettre de s'adapter à un environnement en perpétuel changement. Se pose alors la question de savoir si les trois facteurs qui, depuis la Deuxième Guerre mondiale, ont facilité le progrès des PME japonaises permettront à celles-ci d'affronter les défis actuels, et notamment celui des délocalisations.

Parmi les trois facteurs, la sous-traitance est celui sur lequel les PME ne pourront plus compter. Pour comprendre les raisons de ce changement, il est nécessaire de le placer dans le contexte actuel de délocalisation industrielle. La délocalisation est un processus qui touche tous les pays développés. Toutefois, le Japon, en raison de sa proximité à la fois géographique et culturelle avec l'Asie du Sud-Est est peut-être le pays le plus affecté par ce processus. Ainsi, en 2002, environ $33 \%$ des entreprises industrielles japonaises possédaient des filiales à l'étranger, dont la moitié en Asie (METI, 2003). Évidemment, les petites entreprises industrielles sont les premières affectées. Depuis quelques années, les plus grandes entreprises n'ont plus besoin de leurs soustraitants traditionnels. Les sous-traitants sont menacés par l'investissement et l'approvisionnement à l'étranger de leurs « entreprises-parents », mais aussi par leurs efforts de restructuration et de réduction des coûts. Selon les estimations gouvernementales, le volume d'ordres placés auprès des sous-traitants japonais par les grandes entreprises a en effet baissé de $50 \%$ à $75 \%$ depuis le milieu des années 1990 (METI, 2003).

Le transfert de connaissances et la création de compétences dans le futur ne pourront plus avoir lieu dans le cadre de relations parent-enfant. Par conséquent, les PME vont devoir se baser sur les autres facteurs 
pour développer leurs compétences. La création de compétences pour les PME reposera donc sur leurs forces internes ainsi que sur leurs échanges avec les autres entreprises, noués au sein des districts industriels.

Aux deux sources d'acquisition des compétences restantes, le gouvernement souhaite ajouter la troisième, celle des partenariats avec les institutions de recherche. Ces partenariats constituent le vecteur principal des politiques de création de clusters industriels, tendance que l'on peut également observer en Europe et notamment en France avec les programmes de développement des « pôles de compétitivité ».

Le gouvernement japonais a entrepris des actions pour répondre au phénomène de délocalisation et aider les entreprises à trouver des nouvelles directions de développement. En s'appuyant sur les travaux de Porter (1990, 1998) sur les clusters et les avantages régionaux ainsi que sur sa recommandation selon laquelle le Japon devrait passer du paradigme Doing things better au paradigme Doing different things (Porter et al., 2000), le gouvernement japonais a entamé des actions de développements des clusters - agglomérations d'entreprises de haute technologie, caractérisées par des échanges intenses en connaissance à travers des réseaux. Il s'agit donc d'un phénomène proche de celui des districts industriels. Le projet des clusters peut en quelque sorte être vu comme une tentative de transformation des districts industriels en clusters hi-tech.

Deux ministères japonais ont chacun développé leur projet de clusters. Le METI a développé le projet de développement de clusters industriels (Industrial Cluster
Plan) et le MEXT le projet de clusters de connaissances (Knowledge Cluster Plan). La mise en œuvre du premier a débuté en 2001 et celle du second en 2002. Le projet de METI place les entreprises en tant qu'acteurs centraux des clusters. Le projet de MEXT accentue le côté académique et accorde une place centrale aux universités et centres de recherche. Toutefois, les principes sont très similaires. Il s'agit en fait de financer des projets d'innovation joints des entreprises et des chercheurs. Le point central des projets de clusters est donc la promotion de la coopération en innovation entre les centres de recherche et l'industrie et les PME industrielles sont particulièrement visées.

Se pose alors la question de savoir si des concentrations industrielles en haute technologie peuvent être générées par des politiques. Jusqu'à présent, la naissance des agglomérations industrielles à travers le monde a plutôt été engendrée par les mouvements spontanés des acteurs, autrement dit par 1'auto-organisation. Bien que les politiques puissent stimuler les entreprises et les chercheurs à créer des partenariats afin d'obtenir des financements publics pour leurs projets, il n'est pas certain qu'audelà de la durée des programmes gouvernementaux ces partenariats perdureront.

Pour l'instant de nombreux obstacles, à la fois du côté des entreprises et du côté des chercheurs, empêchent la prolifération de tels partenariats, ces obstacles ayant essentiellement pour origine le peu de liens entres les PME et les institutions publiques de recherche. Un des enjeux des années à venir sera de surmonter ces obstacles et ainsi de permettre aux PME japonaises d'évoluer. 


\section{CONCLUSION}

Cet article a étudié les transformations qu'ont connues les PME japonaises au cours des cinquante dernières années. L'acquisition des compétences se trouve au cœur de celles-ci. Elle a été facilitée par la sous-traitance pour les grandes entreprises, les caractéristiques inhérentes aux PME comme l'esprit entrepreneurial ou la volonté de toujours se dépasser et la concentration dans des districts industriels au sein desquels les entreprises s'engagent dans des activités coopératives à travers des réseaux. La sous-traitance cessant d'être une source d'acquisition des compétences, les forces des PME reposent alors sur les caractéristiques des entrepreneurs et sur la coopération au sein des districts industriels. Les partenariats avec les institutions de recherche de proximité pourront devenir une source d'apprentissage complémentaire. La création de la connaissance au sein des agglomérations industrielles devient alors cruciale pour l'économie japonaise. Elle représente un sujet qui mérite d'être étudié tant par les chercheurs que par les praticiens. En effet, l'expérience japonaise dans ce domaine peut s'avérer utile pour les autres pays confrontés au problème de la délocalisation industrielle.

\section{BIBLIOGRAPHIE}

Aoki M., Information, Incentives, and Bargaining in the Japanese Economy, Cambridge University Press, New York, 1988.

Asanuma B., "Manufacturer-Supplier Relationships in Japan and the Concept of Relationship-Specific Skill”, Journal of the Japanese and International Economies, $\mathrm{n}^{\circ}$ 3, 1989, p. 1-30.

Becattini G., Mercato e forze locali: il distretto industriale, Il Mulino, Bologna, 1987.

Benko G., Lipietz A. (eds.), Les régions qui gagnent - Districts et réseaux: les nouveaux paradigmes de la géographie économique, PUF, Paris, 1992.

Benko G., Lipietz A. (eds.), La richesse des régions. La nouvelle économie géographique, PUF, Paris, 2000.

Carillon J-P., Le " juste à temps » dans la gestion des flux industriels, Éditions d'Organisation, Paris, 1986.

Colovic A., Tayanagi E., "What direction should the cluster policy take - Top-down implementation or bottom-up emergence? The Case of Japan”, Uddevalla Symposium 2003: Entrepreneurship, Spatial Industrial Clusters and Inter-Firm Networks, Research reports University of Trolhattan/Uddevalla, Uddevalla, 2004, p. 319-337.

Dore R., Flexible Rigidities: Industrial Policy and Structural Adjustment in the Japanese Economy, 1970-80, Athlone, London, 1986.

Dore R., Taking Japan Seriously, Athlone, London, 1987.

Fruin M.W., The Japanese Enterprise System, Clarendon Press, Oxford, 1992.

Gerlach M. L., "The Japanese Corporate Network: A Block Model Analysis", Administrative Science Quarterly, vol. 37, n 1, 1992, p. 105-139. 
Hashimoto J., "Keizai, sangyo kozo no tenkan” (traduction : «Changements dans l'économie et dans la structure industrielle ») Gendai shitauke kigyoron (traduction : Les perspectives des firmes sous-traitantes modernes), Kokumin Kinkyu Koko (ed.), Chusho kigyo risachi senta, Tokyo, 1989.

Hay E.J., The Just-in-Time Breakthrough, John Wiley \& Sons Ltd., New York, 1988.

Itami H., Nihon sangyo mitsu no nami (traduction : Les trois vagues de l'industrie japonaise), NTT Press, Tokyo, 1998.

Ito T., The Japanese Economy, MIT Press, Cambridge, Massachusetts and London, England, 1992.

Ito K., Rose E.L., "The Genealogical Structure of Japanese Firms: Parent-Subsidiary Relationships", Strategic Management Journal, vol. 15, special issue, summer 1994, p. 35-51.

Itoh M., Urata S., "Small and Medium-Size Enterprise Support Policies in Japan", Policy Research Working Paper, $\mathrm{n}^{\circ}$ 1403, 1994, The World Bank.

Japanese Small Business Research Institute (JSBRI), "A Study of the Present Conditions and the Future Outlook of Industrial Districts in Japan”, Series no 98-1, 1998.

Kiyonari T., Chusho kigyo tokuhon (traduction : Le lecteur de PME), $2^{\mathrm{e}}$ édition, Toyo keizai shinposha, Tokyo, 1990.

Lung Y., "A New Step in the Evolution of Japanese Industrial Networks?", Japanese Management in the Low Growth Era, Dirks D., Huchet J.-F., Ribault T. (eds.), SpringerVerlag, Berlin, Heidelberg, New York, 1999, p. 229-239.

Matsushima S., "Creation and development of small and medium enterprise policies in postwar Japan”, Entreprises et histoire, n 28, décembre 2001, p. 11-19.

Ministry of Economy, Trade and Industry (METI), Points on the Preliminary Report on the 2003 Basic Survey of Japanese Business Structure and Activities, METI, Tokyo, Mimeo, 2003.

Ministry of Economy, Trade and Industry (METI), The Industrial Cluster Plan, METI, Tokyo, Mimeo, 2002.

Ministry of International Trade and Industry (MITI), "The survey of the industrial districts overview in 1999”, Periodical Press Release, n 59, MITI, Tokyo, 2000.

Ministry of International Trade and Industry (MITI), Small Business White Paper, MITI, Tokyo, 1972.

Ministry of International Trade and Industry (MITI), Economic White Paper, MITI, Tokyo, 1957.

Miwa Y., Firms and Industrial Organization in Japan, Macmillan, Hampshire and London, 1996.

Morris J., Imrie R., Transforming Buyer-Supplier Relationships, MacMillan, Houndmills, Basingstoke and London, 1992.

Nishiguchi T., Strategic Industrial Sourcing, Oxford University Press, Oxford, 1994.

OCDE, Réseaux d'entreprises et développement local, OCDE, Paris, 1996.

OECD, Boosting Innovation: the Cluster Approach, OECD, Paris, 1999. 
OECD, Enhancing SME Competitiveness: The OECD Bologna Ministerial Conference, OECD, Paris, 2001.

Okimoto D.Z., Between MITI and the Market, Stanford University Press, 1989.

Pascale R.T., "Perspectives on Strategy: The Real Story Behind Honda's Success", California Management Review, vol. 26, $\mathrm{n}^{\circ}$ 3, 1984, p. 47-72.

Piore M.J., Sabel C.F., The Second Industrial Divide: Possibilities for Prosperity, Basic Books, New York, 1984.

Porter M., The Competitive Advantage of Nations, Macmillan, London, 1990.

Porter M., "Clusters and the New Economics of Competition", Harvard Business Review, November, 1998, p. 77-90.

Porter M., Sakakibara M., Takeuchi H., Can Japan Compete?, Palgrave Macmillan, Basingstoke, 2000.

Sako M., Prices, Quality and Trust: Inter-firm Relations in Britain and Japan, Cambridge University Press, Cambridge, 1992.

Sato Y. (ed.), Kyodai toshi no reisai kogyo (traduction : Micro-industrie dans les grandes villes), Nihon keizai hyoronsha, Tokyo, 1981.

Schonberger R.J., Gilbert J.P., "Just-in-Time Purchasing: A Challenge for U.S. industry", California Management Review, vol. 26, $\mathrm{n}^{\circ}$ 1, 1983, p. 54-68.

Seki M., Beyond the Full-Set Industrial Structure, Japanese Industry in the New Age of East Asia, LTCB International Library Foundation, Tokyo, 1994.

SME Agency, The White Paper on Small and Medium Enterprises in Japan, MITI, Tokyo, 2000.

SME Agency, The White Paper on Small and Medium Enterprises in Japan, MITI, Tokyo, 2002.

Storz C., "Which Role Does Networking Play in Japanese SMEs? The Importance of Building up Networks in Japan”, Small Business Monograph, $\mathrm{n}^{\circ} 11$, Institute of Small Business Research and Business Administration, Osaka University of Economics, 1999.

US Department of Commerce, Japan, the government-business relationship, US Department of Commerce, 1972.

Whittaker D.H., Small Firms in the Japanese Economy, Cambridge University Press, Cambridge, UK, 1997. 
